\title{
ULTRASONIC MICROINJECTION CHARACTERIZED BY INTEGRATED MICRO-OPTICAL FORCE ENCODER
}

\author{
Xiaojing Zhang, Stefan F. Zappe, Calvin F. Quate, Matthew P. Scott* and Olav Solgaard \\ Edward L. Ginzton Laboratory, Department of Electrical Engineering \\ * Department of Developmental Biology, and of Genetics, School of Medicine \\ Stanford University, Stanford, CA 94305
}

\begin{abstract}
The effect of ultrasonic agitation on the required penetration force for microinjection in Drosophila embryos was studied, using an integrated optical MEMS force encoder for in-vivo characterization of the dynamic penetration forces. Two modes of operation were investigated. In the first mode of operation, the injector was brought into contact with the embryo to give a fixed bias offset of the two encoder gratings, and the vibration amplitude of the microinjector was increased till penetration occurred. We observed a linear decrease in the penetration force of $2.5 \mu \mathrm{N}$ with every $0.1 \mathrm{~m} / \mathrm{s}$ tip velocity increase. In the second mode of operation, the vibration amplitude was kept constant and the injector was pushed into the embryo until penetration. We measured the injection force over the frequency range of $0 \sim 16 \mathrm{kHz}$ with actuation voltage up to 150 volts. The average penetration force was calculated based on injections on multiple embryos for each experimental condition. The achieved minimum peak penetration force was $15.6 \mu \mathrm{N}(\sim 29.7 \%$ of the static penetration force), while the minimum average penetration force was $2.7 \mu \mathrm{N}$ ( $5.1 \%$ of the static penetration force).
\end{abstract}

\section{INTRODUCTION}

Minimally invasive microsurgical tools for localized and accurate trans-membrane delivery of genetic material into biological model systems, such as Drosophila, will enable a variety of studies of developmental biology and genetics. Vibration is a well-known method for reducing the cutting force of macroscopic tools. For microsurgical devices, improved efficiency was first observed in dissecting cataractous lenses using piezoelectricallyactuated, centimeter-scale silicon cutters [1]. The cutting force was later characterized using piezoresistors [2].

Recently, we demonstrated a micro-optical, encoder-based force sensor for characterization of the force required for penetration and injection of Drosophila embryos [3]. Minimization of the penetration force is critical to the design of high throughput micro-manipulating instruments [4,5,6] for biology and genetics studies, such as RNAi for gene silencing [7]. In this paper, we report on penetration force minimization through ultrasonically actuation of silicon-nitride microinjectors. Actuated by piezoelectric actuators, the injectors are longitudinally vibrated with tip velocities controllable by actuation frequency and voltage. The forces are measured using MEMS optical-encoder force sensors integrated with the vibrating microinjectors.

\section{THEORY}

Ultrasonic microinjection was performed with silicon-nitride probes vibrated by piezoelectric actuators, as shown in Figure 1. The probes have integrated force sensors consisting of two vertically-separated microgratings. With no force applied, the two gratings are perfectly aligned. The upper index grating is connected to the microinjector, and has a resonant frequency of $\mathrm{f}_{0}=14 \mathrm{KHz}$. The static diffraction characteristics of the force encoder under normal plane-wave illumination can be analyzed by Fraunhofer diffraction theory [9].

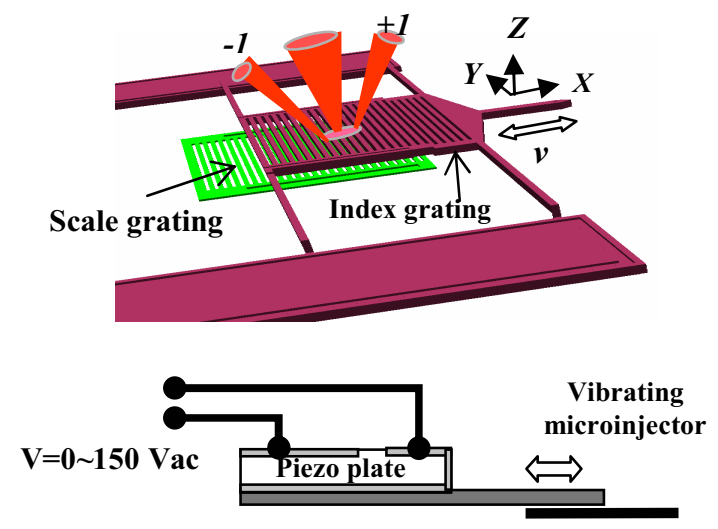

Figure 1. Ultrasonic microinjection characterized by integrated optical MEMS force encoder. A piezoelectric stack is bonded onto the backside of the force sensor chip. The probe displacement at maximum actuation voltage of $150 \mathrm{~V}$ is $17.4 \mu \mathrm{m} \pm 2.0 \mu \mathrm{m}$.

The first diffraction mode intensity $I_{l}$ is a periodic function of injector displacement $d(t)$, as shown by the solid curve in Figure 2. Under ultrasonic actuation, the index grating vibrates along with the microinjector and hence changes its position relative to the fixed scale grating. The force on the injector is determined by the relative displacement $d(t)$ of the two gratings, which is determined by the intensity distribution of the diffraction orders. For the first diffraction mode, we have:

$\left.I_{1}[d(t)]=I_{0} \cdot N^{2} \cdot\left[\frac{\sin c^{2} \frac{N \cdot d(t)}{2 L}}{\sin ^{2} \frac{d(t)}{2 L}}\right] \cdot\left\{[L-d(t)] \cdot \sin c \frac{[L-d(t)]}{4 L}\right\} \cdot \sin ^{2} \phi_{0} \cdot G d(t)\right]$

where $I_{0}$ is the illuminating light intensity, $N$ is the number of grating periods under illumination, $\phi_{0}(x)=(2 \pi / \lambda) \cdot\left(n_{1}-n_{0}\right) \cdot t$ is the phase-delay over the thickness of one grating finger, $2 L$ is the period of the grating, $G[d(t)]=\sin ^{2}\{\pi[L+|d(t)-L|] / 4 L\}$ is phasemodulating term, $d_{0}(t)$ is the quasi-static relative displacement of the two gratings caused by the linear motion of the microinjector relative to the target, $d_{V}(t)$ is the sinusoidal displacement of the injector under piezoelectric actuation. The relative displacement can be expressed:

$$
d(t)=d_{0}(t)+d_{V}(t)
$$

Travel support has been generously provided by the Transducers Research Foundation and by the DARPA MEMS and DARPA Bioflips programs 
where

$$
d_{V}(t)=A \cdot \sin \left(\omega_{P} \cdot t+\varphi_{P}\right)=\alpha \cdot V_{a} \cdot \sin \left(2 \pi \cdot f_{P} \cdot t+\varphi_{P}\right)
$$

and $\alpha=0.116 \mu \mathrm{m} / \mathrm{V}, V_{a}, f_{p}$ are the piezoelectric constant, the actuation voltage, the driving frequency of the piezo-actuator, specifically. The force is related to the displacement through the spring constant, $k_{x}$, of the encoder:

$$
F=k_{x} \cdot d(t)
$$
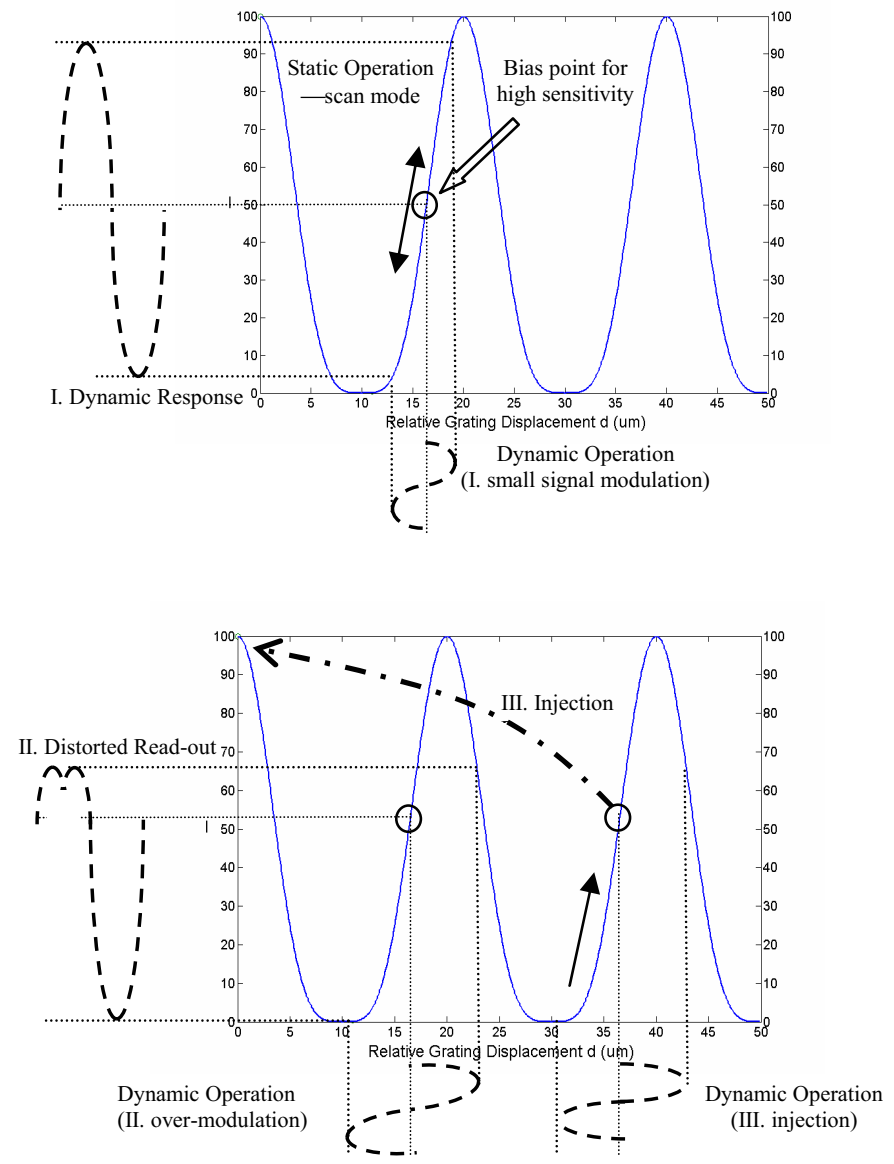

Figure 2. The sensor output evolves from a sinusoidal signal at small injector displacement (top), to a distorted output as the tip velocity increases (bottom). After penetration, the encoder bias point recovers to zero and the output signal doubles in frequency.

The penetration of the embryo membrane can be caused by either linear or vibration motion of the microinjector. We assume the microinjector interacts with the membrane during the full vibration cycles till penetration. In our studies, the linear translation velocity of the microinjector is much smaller than that of the vibration. The average (or peak) penetration force can be derived from the average (or peak) relative displacement of the two gratings. The measured spring constant of encoder $k_{x}$ is $1.85 \mathrm{~N} / \mathrm{m}$.

The dotted lines in Fig. 2 show how the force sensor output evolves from a sinusoidal signal at small injector displacement (case I), to a distorted signal (case II) as the injector vibration amplitude $A$ increases. Penetration, caused by either increased vibration or translation, significantly reduces the force on the injector. Consequently, the average relative grating displacement goes to zero, and the output signal abruptly changes to a periodic signal with doubled frequency (case III).

\section{DESIGN AND FABRICATION}

The force encoders were fabricated by LPCVD deposition and patterning of two silicon nitride layers for the dual gratings, separated by low temperature sacrificial oxide, on the silicon substrates. The fabrication process is described in detail in [8].

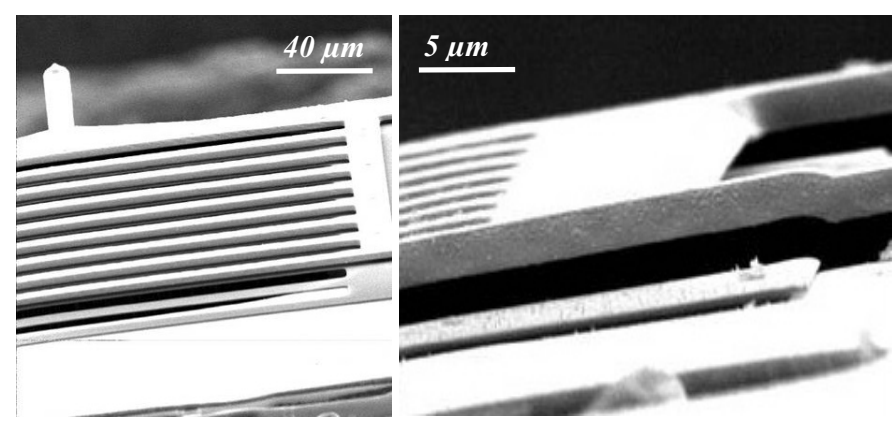

Figure 3 Scanning electron micrographs show the force probe integrated with the movable index grating (left), and 3 rm uniform vertical gap between the dual gratings (right).

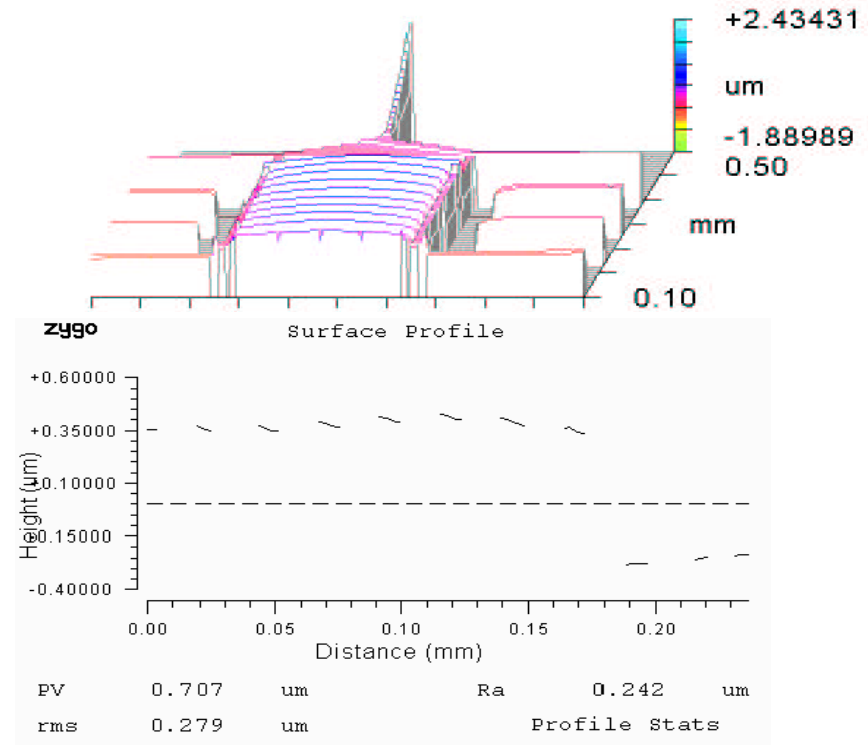

Figure 4 Movable index grating optical surface profile with roughness $50 \mathrm{~nm}$ peak-to-peak and uniformity 1.25\% (data acquired using Zygo white-light 3D surface profiler).

Scanning electron micrographs (SEMs) of the micromachined optical encoder force sensor, with large vertical gaps suitable for ultrasonic actuation, are shown in Fig. 3. The SEMs show the force probe that is integrated with the movable index grating, the index and scale gratings with $20 \mu \mathrm{m}$ pitch, and a close-view of the $3 \mu \mathrm{m}$ vertical gap and junction between the gratings and the supporting beams.

The silicon nitride grating layers, deposited under $\mathrm{NH}_{3}$-rich conditions, have excellent optical surface quality. Figure 4 shows the surface topography characterization results of the encoder gratings using optical phase-shifting interferometry (Zygo whitelight $3 \mathrm{D}$ surface profiler). The index grating surfaces show less than $50 \mathrm{~nm}$ root-mean-square roughness. Note that the overhanging probe appears to be bending upwards. This is caused 
by the shift of optical reference plane due to the removal of the silicon substrate under the probe.

The sensor chip is completed by bonding a piezoelectric stack, consisting of many piezoelectric ceramic layers that are assembled in series mechanically and in parallel electrically, onto the back side of the chip (Fig. 1). The piezoelectric stack is used to vibrate the microinjector longitudinally in the $X$-direction. The piezo-actuator has a resonance frequency of $69 \mathrm{kHz}$, and the displacement at the maximum drive voltage of 150 volts is 17.4 $\mu \mathrm{m} \pm 2.0 \mu \mathrm{m}$.

\section{EXPERIMENTAL RESULTS AND DISCUSSION}

The measurement set-up for ultrasonic microinjection and invivo penetration force characterization is shown in Figure 5.

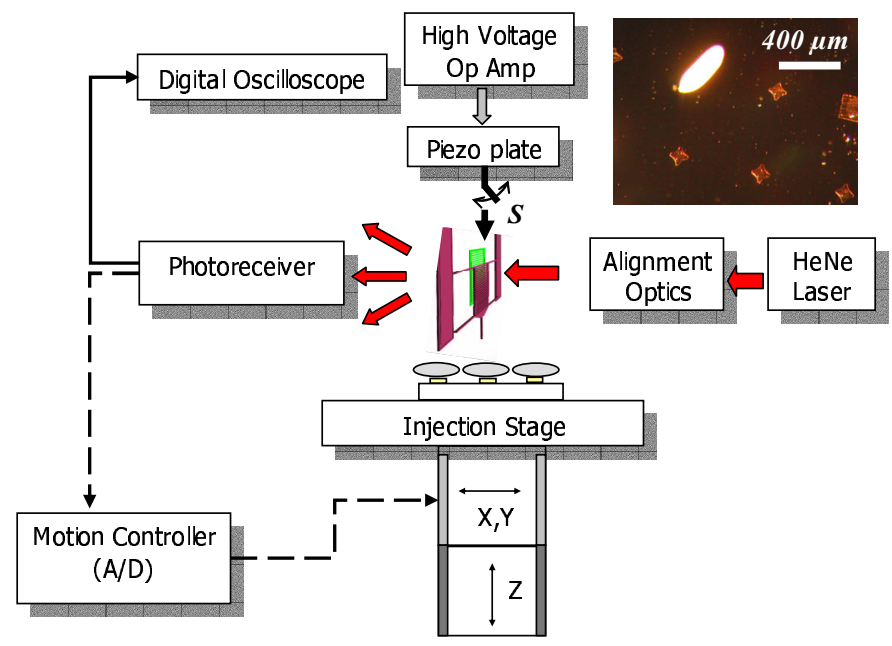

Figure 5. Experimental set-up for operating optical encoder force sensor on Drosophila embryos positioned on 2-D self-assembly chip(shown in the embedded optical micrograph). The power in the first-order diffracted mode is measured with a photo detector.

Newly hatched Drosophila embryos [10] were positioned on 2-D micro-fluidic self-assembly chip [6] ready for vertical injection. The encoder was illuminated by a $\mathrm{HeNe}$ laser $(633 \mathrm{~nm} / 4 \mathrm{~mW})$ with spot sizes ranging from 60 to $160 \mu \mathrm{m}$ to achieve tunable sensitivity, and the output intensity signals were detected using a photoreceiver connecting to an oscilloscope. Spatial filtering was preformed to minimize the cross-talk between diffraction orders and therefore optimize the contrast. In earlier static penetration measurements (switch $S$ open), we found an average penetration force of $52.5 \pm 13.2 \% \mu \mathrm{N}$ and an embryo deformation of $58 \pm 5.2 \% \mu \mathrm{m}[3]$.

During ultrasonic microinjection experiments ( $S$ closed), the injector is translated towards the target while vibrating. The indexgrating vibrates along with the injector and hence changes its position relative to the fix scale grating. The force acting on the injector is determined by the relative displacement of the two gratings, which is determined by the intensity distribution of the first diffraction order under observation.

Two sets of ultrasonic microinjection experiments were performed. First the injector was brought into contact with the embryo to give a fixed bias offset of the two gratings. At each bias point, the actuation voltage, and therefore the injector tip velocity, was increased till penetration $\left(f_{p}=14 \mathrm{kHz}\right)$. The critical peak-topeak actuation voltage $V_{a}$ for penetration can be used to calculate probe vibration amplitude $d_{y}=\alpha \cdot V_{a}$ and tip velocity $v_{p}=4 \cdot d_{y} \cdot f_{p}$.

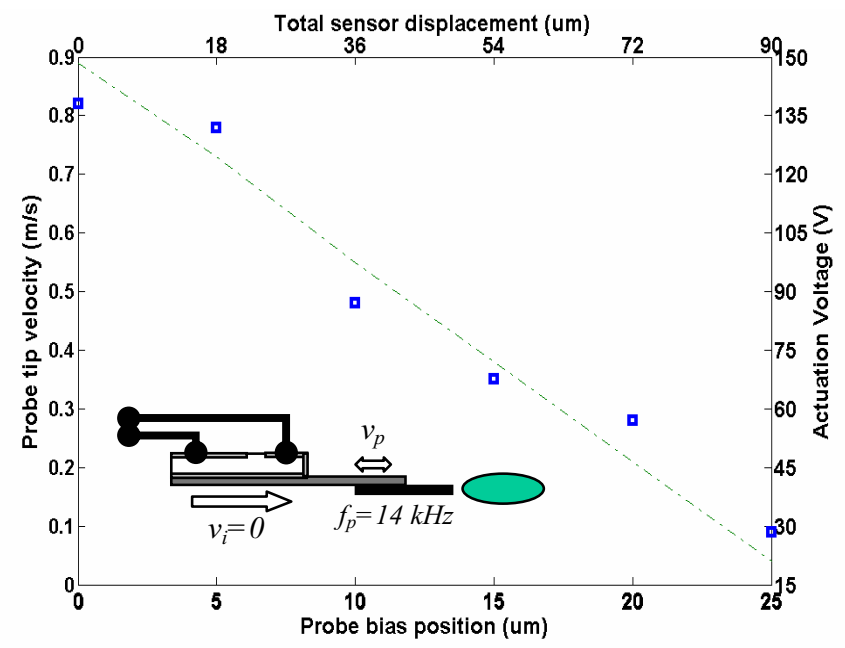

\begin{tabular}{ccc|cc}
\hline $\boldsymbol{d}_{\boldsymbol{0}}(\boldsymbol{\mu \mathbf { m }})$ & $\boldsymbol{V}_{\boldsymbol{a}}(\mathbf{V})$ & $\boldsymbol{d}_{\boldsymbol{v}}(\boldsymbol{\mu} \mathbf{m})$ & $\boldsymbol{v}_{\boldsymbol{p}}(\mathbf{m} / \mathbf{s})$ & $\mathbf{F}_{\mathbf{p}}(\boldsymbol{\mu} \mathbf{N})$ \\
\hline 0 & 126 & 14.64 & 0.82 & 27.08 \\
5 & 120 & 13.93 & 0.78 & 35.02 \\
10 & 74 & 8.57 & 0.48 & 34.35 \\
15 & 54 & 6.25 & 0.35 & 39.31 \\
20 & 43 & 5.00 & 0.28 & 46.25 \\
25 & 14 & 1.61 & 0.09 & 49.23 \\
\hline Table 1 & Peak penetration force & $F_{p}$ & versus bias position $d_{0}$,
\end{tabular}
actuation voltage $V_{a}$, vibration amplitude $d_{y}$ and tip velocity $v_{p}$.

Figure 6. Experimental results for ultrasonic microinjection into Drosophila embryos. The injector was pushed into the embryo till a fixed bias point. For each bias point, the actuation voltage, and therefore the injector tip velocity, was increased $\left(f_{p}=14 \mathrm{kHz}\right)$ till penetration occurred. A linear relationship is found between probe tip velocity at penetration and probe bias position, showing the peak penetration force decreasing by $\sim 2.5 \mu N$ with every $0.1 \mathrm{~m} / \mathrm{s}$ tip velocity increase.

As shown in Fig. 6, with zero static force bias, the minimum tip velocity needed for penetration is $v_{p}=0.82 \mathrm{~m} / \mathrm{s}$ (actuated at $f_{p}=14 \mathrm{kHz}$ and $V_{a}=126 \mathrm{~V}$ ). This corresponds to a peak penetration force of $27.1 \mu \mathrm{N}$, or $48.5 \%$ reduction compared to the static penetration without vibration. Table 1 summarizes the calculated tip velocity and the associated peak penetration force at each fixed bias offset of the microinjector. In a series of experiments, we observed a linear decrease in the penetration force of $\sim 2.5 \mu \mathrm{N}$ with every $0.1 \mathrm{~m} / \mathrm{s}$ tip velocity increase.

In the second set of experiments, the vibration amplitude was kept constant and the injector was pushed into the embryo until penetration. Both peak and average penetration force were measured. Figure 7 shows the measured peak penetration force as a function of actuation voltage and frequency. Each of the 170 points on the surface represents an averaged peak penetration force measured on 3 to 5 embryos. The read-out of the total linear injection-stage displacement upon penetration is scaled with respect to the encoder pitch period to get the linear displacement $d_{0}$ between the two gratings, while the amplitude of microinjector vibration can be calculated based on the actuation frequency and voltage. The peak force can then be derived from the total relative displacement of the two gratings.

Both the average and the peak penetration force decreases as the driving frequency and/or actuation voltage increases. At fixed actuation voltage, the penetration force reached a minimum at the encoder resonant frequency of $14 \mathrm{kHz}$. At the maximum actuation 
voltage of $150 \mathrm{~V}$, the minimum tip velocity for penetration was $0.96 \mathrm{~m} / \mathrm{s}$. The minimum peak penetration force was $15.6 \mu \mathrm{N}$, $29.7 \%$ of the static penetration force, while the minimum average penetration force was $2.7 \mu \mathrm{N}, \sim 5.1 \%$ of the static penetration force.

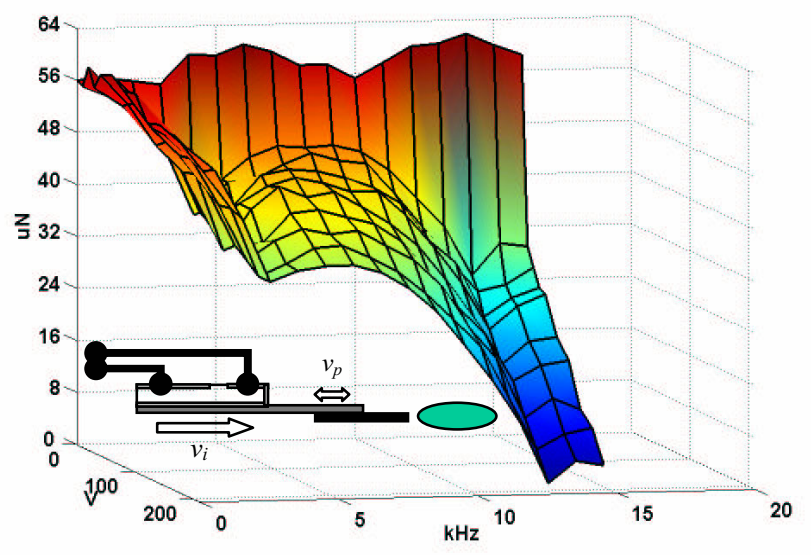

Figure 7. The peak penetration force was measured over the frequency range $f_{i}=0 \sim 16 \mathrm{kHz}$ with the actuation voltage $V_{j}$ varying from 0 to 150 volts. The average force was calculated based on injections on multiple embryos under each of the 170 experimental conditions $\left(f_{i}, V_{j}\right)$. The penetration force reached its minimum at the encoder resonant frequency of $14 \mathrm{kHz}$.

\section{CONCLUSIONS}

Minimally invasive microsurgical tools with integrated sensors are critical for a wide range of studies in biology and medicine, including calibrated trans-membrane delivery of genetic material into biological model systems, such as Drosophila embryos, to enable high throughput screening of gene functions. We demonstrated penetration force minimization through ultrasonically actuation of silicon-nitride microinjectors. The forces are measured using MEMS optical-encoder force sensors integrated with the microinjectors. Significant force reduction through dynamic tip-membrane interaction was achieved. For vibration-only operation, the force is reduced by $4.8 \%$ for every $0.1 \mathrm{~m} / \mathrm{s}$ velocity increase. When the motion of the force probe is a combination of vibration at the encoder resonance and linear translation, the peak force is reduced by $70.3 \%$, while the average force is reduced by $94.9 \%$ with respect to the force required for linear translation alone.

\section{ACKNOWLEDGEMENTS}

This project was funded by DARPA [Bio:Info:Micro] program (MDA972-00-1-0032). The device fabrication and characterization were performed at the National Nanofabrication Users Network (NNUN) facilities at the Center for Integrated systems and Edward L. Ginzton Lab at Stanford University. The authors wish to thank Matthew Fish for preparing Drosophila embryos for microinjection experiments.

\section{REFERENCES}

[1] A. Lal and R.M. White, "Micromachined Silicon Needle for Ultrasonic Surgery", Proc. of IEEE Ultrason. Sym., 1593-95, 1995.

[2] A. Lal, "Silicon-based Ultrasonic Surgical Actuators", Proc. of the $20^{\text {th }}$ Annual International Conference of the IEEE Engineering in Medicine and Biology Society, Piscataway, NJ, USA, 2785-90, vol. 6, 1998.

[3] X.J. Zhang, S. Zappe, R.W. Bernstein, O. Sahin, C.-C. Chen, M. Scott and O. Solgaard, "Integrated Optical Diffractive Micrograting-based Injection Force Sensor", Proc. of the International Conference on Solid State Sensors and Actuators, p1051-1054, Boston, USA, 2003.

[4] S. Zappe, X.J. Zhang, I.W. Jung, R.W. Bernstein, E. Furlong, M. Fish, M. Scott and O. Solgaard, "Micromachined Hollow Needle With Integrated Pressure Sensor For Precise, Calibrated Injection Into Cells and Embryos", Proc. of the International Symposium on Miniaturized Chemical and Biochemical Analysis Systems, vol.2, p682-684, November 3-7, Nara, Japan, 2002.

[5] R.W. Bernstein, X.J. Zhang, S. Zappe, M. Fish, M. Scott and O. Solgaard, "Characterization of Drosophila Embryos Immobilized by Fluidic Microassembly", Proc. of the International Conference on Solid State Sensors and Actuators, vol. 2, p987-990, June8-12, Boston, USA, 2003

[6] X.J. Zhang, C.C. Chen, R.W. Bernstein, S. Zappe, M.P. Scott and O. Solgaard, "Micro-optical Detection and Modeling of Microfluidic Self-assembly in Biology", Journal of Microelectromechanical Systems (in review).

[7] P.A. Sharp and P.D. Zamore, "RNA Interference", Science 287, 2430, 2000

[8] X.J. Zhang, S. Zappe, R.W. Bernstein, O. Sahin, C.-C. Chen, M. Scott and O. Solgaard, "Micromachined Silicon Force Sensor Based on Diffractive Optical Encoders for Characterization of Microinjection", Sensors and Actuators, Phy. A, in press, appeared online on January 22, 2004.

[9] J.W. Goodman, Introduction to Fourier Optics, $2^{\text {nd }}$ ed., 1996.

[10] Drosophia embryos 50 minutes after hatching are dechlorinated in $60 \%$ bleach for 1.5 minutes and then rinsed thoroughly with water $\left(20^{\circ} \mathrm{C}\right)$. Properly staged embryos are selected and desiccated for $15 \mathrm{~min}$ in a sealed glass jar containing calcium sulfate $\left(\mathrm{CaSO}_{4}\right)$ desiccant. Finally, embryos are covered in Halocarbon 700 oil (Aqua-Air Industries Inc., Harvey, LA) and ready for microinjection. 